\title{
Spinal cord injury after high-voltage electrical burns
}

Liubov Adrover Kirienko, Alejandra Monte Soldado, Maria Lluïsa Torrent Bertran M.D., Mireia Ruiz Castilla M.D., Miguel Ángel González Viejo M.D

Physical Medicine and Rehabilitation \& Plastic and Reconstructive Surgery Departments of Vall d' Hebron Univesity Hospital, Barcelona, Spain.

Introduction : Spinal cord injury is a rare complication following high voltage electrocution ( $>1000$ volts), that can be immediate or delayed (daysweeks after the accident). In these cases, intramedullary signal abnormalities can be found in a Magnetic Resonance Imaging (MRI), usually described as a hyperintensity signal in $\mathrm{T} 2$ sequences, although some patients present normal MRI.

Objective: To describe delayed spinal cord injury characteristics and diagnosis after high-voltage electric shock without vertebral fracture.

Methods: We report 2 cases of patients who received inpatient care at the Burn Unit of Vall d' Hebron University Hospital in Barcelona (Spain), both with spinal cord impairment and no vertebral injury associated, secondary to electrical accident.

Case 1: 17 years old male suffers a high-voltage electrocution after handling an electrical power transformer.
Case 2: 28 years old male old male suffers a high-voltage electrocution after handling electrical wiring.

Medical History: heroine ex-consumer

- Medical History: hypersensitivity to metamizol, cannabis active smoker, psychotic disorder on treatment.

- Physical examination: 3rd degree burns in $18 \%$ of total surface body area (TBSA) affecting upper and lower extremities with circular ones affecting the arms. Exit point on right forearm and bilateral claw hand deformity.

- Immediate complication: upper limbs muscular necrosis that required bilateral transhumeral amputation.

- Day 10: acute urinary retention and bilateral lower extremities weakness. Diagnosis by neurological examination: sensory-motor incomplete Epiconus Syndrome (grade C in ASIA scale).

- Day $66^{\circ}$ : entrance to the Spinal Cord Injury Unit for Rehabilitation Intensive Program.
Physical examination: 2nd-3rd degree burns in 7\% of TBSA affecting face, occiput, both arms and right feet. Entry point on the left hand, exit point on the occiput and left claw hand deformity.

Immediate complication: left forearm compartment syndrome that required urgent fasciotomy.

- Day 80: bilateral lower extremities weakness. Diagnosis by neurological examination: incomplete sensory-motor dorsal spinal cord injury, neurological preserved level D7 (grade C in ASIA Scale).

- Day 10: transradial left amputation.

- Day 650 : entrance to the Spinal Cord Injury Unit for Rehabilitation Intensive Program

- None of the patients had spinal cord damage or vertebral fractures at MRI of dorso-lumbar spine. Medullary MRI was no repeated.

- Cranial and spinal CT showed no injury.

- Both patients presented alterations of pyramidal path and somatosensory evoked potentials in Neurophysiological exam.
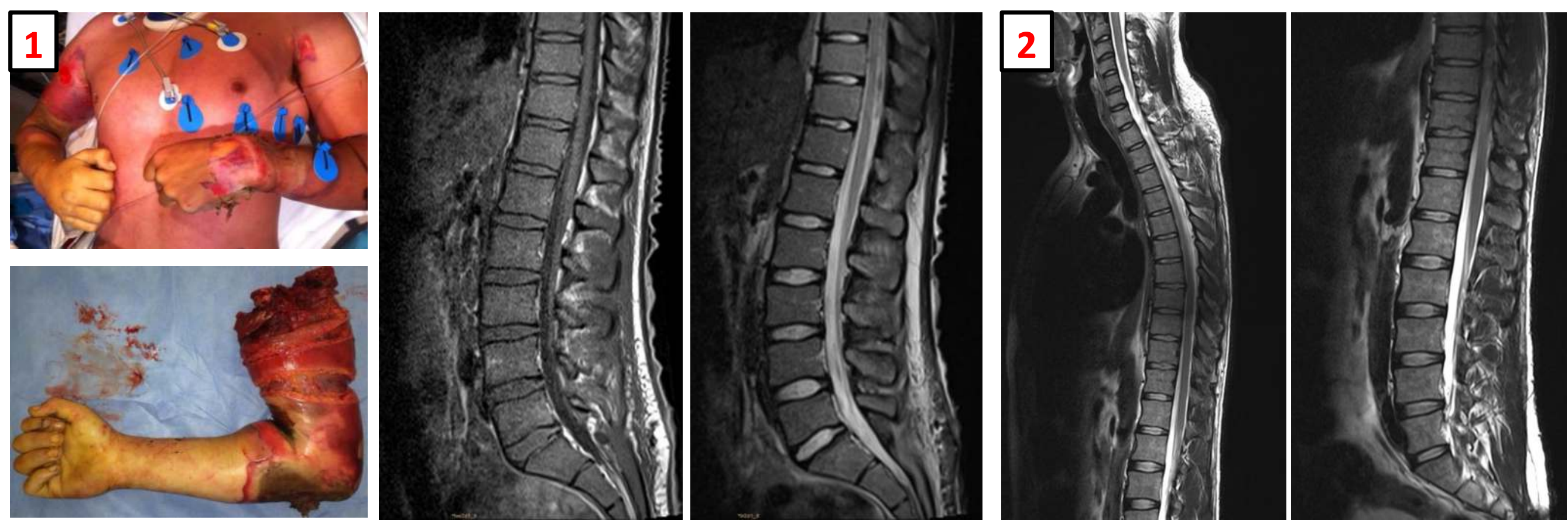

Results: During patients' hospital stay in both, the Burn Care Unit and Spinal Cord Injury Unit, they received Physical and Occupational Therapy treatment in order to avoid skeletal deformities, recover sitting and standing balance, train basic life activities and recover strength and gait capacity. After rehabilitation program, both patients improved in their ASIA spinal cord injury classification grade but still presented important functional sequelae.

\section{Outcome Case 1}

After rehabilitation treatment, patient was discharged with final diagnose of Epiconus syndrome grade D in ASIA scale, achieving independent gait capacity, but total dependence for daily living activities (ADL's) performance secondary to intolerance of upper limb myoelectric prosthesis.

\section{Outcome Case 2}

After rehabilitation treatment, the patient was discharged with final diagnose of Incomplete sensorymotor spinal cord injury preserved level D7 grade D of ASIA scale, achieving independent crutch-assisted gait capacity and partial dependence for ADL's performance.

\section{ASIA Impairment Scale (AIS)}

$A=$ Complate tha sersory ar motrs finction is presered the soud segments $\mathrm{S4}-5$.

$\mathrm{B}=$ Sensary incomplete. Sensary bat nar motor function is presened below the neurblogkal levil and holudes the sacal segments S4-5 figh buch or ph prok $a$ S4-5 or deep and

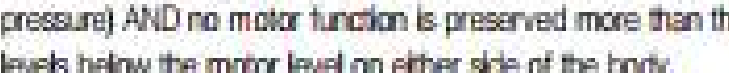

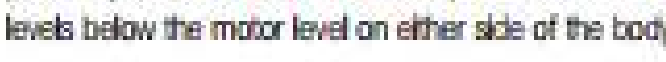

$\mathrm{C}=$ Motor Incomplete. Motar functon is presened at the

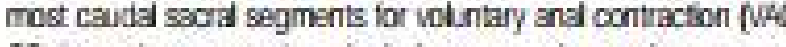

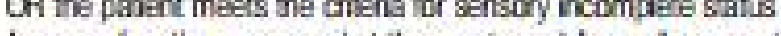

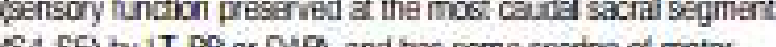
(64-S5) by LT, PP or DAP, and has some spang of mota

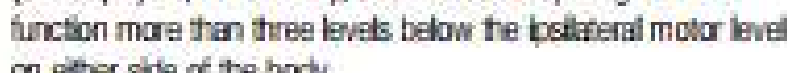
on either side of the body.

(This inclides kej or non-key muscle tunctons ta determine motor hoomplete satus) For ASC - less then hat of hey musda tuncions below the ingle RLi have a miscie gode a 3

D = Motor Incomplete. Motor incomplat stas as define above with a least hat that or madel of key misde tunctons

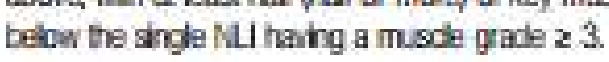

$E=$ Normal. If sensaibn and motor tuncion as festad wi

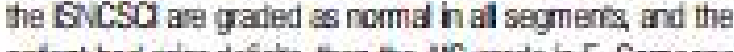
palent had prar deicha then the AS gade is E. Sombone

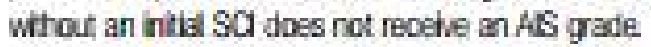

Using ND: To doument the serens motr and Nu bek the ASA mpdiment Scale gade, andor the mone of partial resenation (2PP) when they are unath ta be determined cased on the examintibn resil:

\section{SiNA}

NTERNATONAL STANDARDS FOR MEUROLOGICAL CLASSIFICATION OF SPINAL CORD INJURY ISCS

Conclusion: Spinal cord injury following high-voltage electrocution is an infrequent complication but needs an early diagnosis and multidisciplinary approach given the severity of sequels and the immense impact on their functionality, autonomy and participation in the Society .

Sources:

1)Delayed myelopathy following lightning strike: a demyelinating process. Acta Neuropathol. 1988;77(1):104-8.; 2)MR imaging findings in delayed reversible myelopathy from lightning strike. AJNR Am J Neuroradiol. 2004 May;25(5):851-3; 3)Neuropathology of lightning-strike injuries.Semin Neurol. 1995 Dec;15(4):323-8. Review. 4)High voltage electrical injury leading to a delayed onset tetraplegia, with recovery. Ann N Y Acad Sci. 1999 Oct 30; 888:131-6.; 5)Delayed neurologic injury secondary to high-voltage current, with recovery. J Trauma. 1980 Feb;20(2):166-8, 6)Cervicothoracic spinal cord and portomedullary injury secondary to high-voltage electrocution: a case report. J Med Case Rep. 2012 Sep 13;6:296.; 7) Neurorehabilitation of spinal cord injuries following lightning and electrical trauma. NeuroRehabilitation. 2005;20(1):9-14.; 8) The Spinal Cord in Lightning Injury: A Report of Two Cases. Neurol Sci 276 (1-2), $199-201.2008$ Oct 23. 